\title{
Atividade antileishmania do extrato hidroalcoólico e de frações obtidas de folhas de Piper regnellii (Miq.) C. DC. var. pallescens (C. DC.) Yunck
}

\author{
Celso V. Nakamura, Adriana O. Santos, Michele C. Vendrametto, Patrícia S. Luize, Benedito \\ P. Dias Filho, Diógenes A.G. Cortez, Tânia Ueda-Nakamura*
}

Programa de Pós-Graduação em Ciências Farmacêuticas, Universidade Estadual de Maringá, Av. Colombo, 5790, 87020-900, Maringá, PR, Brasil

\begin{abstract}
RESUMO: A atividade biológica do extrato bruto e as várias frações obtidas de folhas de Piper regnellii (Miq.) C. DC. var. pallescens (C. DC.) Yunck foi avaliada em Leishmania amazonensis. Este estudo incluiu o processo de extração e o fracionamento biomonitorado pelo método de cromatografia de adsorção. Um aumento progressivo no efeito antileishmania foi observado durante o processo de purificação. O extrato hidroalcoólico solúvel em água (EBA) apresentou concentração inibitória $50 \%\left(\mathrm{IC}_{50}\right)$ igual a $167 \mu \mathrm{g} / \mathrm{mL}$ enquanto o extrato hidroalcoólico solúvel em acetato de etila (EBAcOEt) mostrou um $\mathrm{IC}_{50}$ de $30 \mu \mathrm{g} / \mathrm{mL}$ sobre o crescimento de formas promastigotas após $48 \mathrm{~h}$ de cultivo. A fração hexano (FHex) apresentou uma atividade antileishmania maior que o EBAcOEt com $\mathrm{IC}_{50}$ de $21,5 \mu \mathrm{g} / \mathrm{mL}$. Análises de citotoxicidade indicaram que as concentrações tóxicas do EBA, EBAcOEt e das frações foram maiores para macrófagos J774G8 do que para os protozoários.
\end{abstract}

Unitermos: Piper regnellii, Leishmania amazonensis, pariparoba, atividade antileishmania, promastigota.

\begin{abstract}
Antileishmanial activity of hydroalcoholic extract and fractions obtained from leaves of Piper regnellii (Miq.) C. DC. var. pallescens (C. DC.)”. Biological activity of the crude extract and several fractions obtained from Piper regnellii var. pallescens was assessed on Leishmania amazonensis. This study included the extraction process and bioassayguided fractionation by the adsorption chromatography method. A progressive increase in the antileishmanial effect was observed in the course of the purification process. The hydroalcoholic extract water soluble (EBA) had a 50\% inhibitory concentration $\left(\mathrm{IC}_{50}\right)$ at $167 \mu \mathrm{g} / \mathrm{mL}$ whereas the hydroalcoholic extract acetate soluble (EBAcOEt) showed an $\mathrm{IC}_{50}$ of $30 \mu \mathrm{g} / \mathrm{mL}$ against the growth of promastigote forms after $48 \mathrm{~h}$ of culturing. The hexan fraction (FHex) showed an antileishmanial activity greater than EBAcOEt with $\mathrm{IC}_{50}$ at $21.5 \mu \mathrm{g} / \mathrm{mL}$. Analysis of cytotoxicity indicated that the toxic concentrations of the EBA, EBAcOEt, and fractions were higher for J774G8 macrophages than for the protozoans.
\end{abstract}

Keywords: Piper regnellii, Leishmania amazonensis, pariparoba, antileishmanial activity, promastigote.

\section{INTRODUÇÃO}

A leishmaniose é uma doença que afeta aproximadamente 12 milhões de pessoas no mundo. A cada ano dois milhões de novos casos são diagnosticados (WHO, 2002). Os agentes causadores dessa doença são parasitas unicelulares heteroxênicos do gênero Leishmania que apresentam duas formas morfológicas no seu ciclo de vida: promastigota e amastigota (Michalick, 2003). As formas promastigotas caracterizam-se por serem fusiformes e com longo flagelo; são extracelulares e vivem no trato digestório do inseto vetor. As formas amastigotas, por sua vez, são arredondadas e com curto flagelo que não se exterioriza; são intracelulares obrigatórias e parasitam principalmente macrófagos do hospedeiro vertebrado.

Essa zoonose compreende formas clínicas que podem ser divididas em dois grupos principais: a forma tegumentar e a forma visceral. A forma tegumentar apresenta variantes e pode ser subdivida em leishmaniose cutânea localizada, cutânea difusa e cutâneo-mucosa. Estas manifestações dependem da espécie de parasita, bem como da resposta imune do indivíduo infectado (Grevelink; Lerner, 1996; Herwaldt, 1999). O tratamento de doenças causadas por esses protozoários é difícil, pois, por serem também eucariotos compartilham muitas 
características com as células de mamíferos. Dessa forma, a atuação dos agentes antiparasitários ocorre em vias ou alvos comuns ao parasita e ao hospedeiro (Murray et al., 2000). Os compostos antimoniais indicados para o tratamento das leishmanioses são o Pentostan ${ }^{\circledR}$ e o Glucantime $^{\circledR}$ (Croft; Coombs, 2003). Como drogas de segunda escolha podem ser utilizadas a Anfotericina B ou a Pentamidina (Grevelink e Lerner, 1996; Sereno et al., 2000). Contudo, vale lembrar que além do alto custo, essas drogas são tóxicas e apresentam muitos efeitos colaterais.

Assim, a medida profilática ideal seria o desenvolvimento de uma vacina. Embora haja muitos grupos trabalhando na tentativa de desenvolvê-la (Convit et al., 2004; Tewary et al., 2004) ainda há vários fatores que dificultam, tais como os mecanismos efetores da resposta imune no homem, além de obstáculos de ordem política e econômica (Handman, 1997). Devido a essas dificuldades, torna-se importante a busca por novos agentes quimioterápicos. Nesse contexto, uma alternativa bastante promissora é o uso de espécies vegetais, o que na verdade já vem sendo utilizado desde o início da civilização no processo de cura de inúmeras doenças (Calixto, 2000; Rocha et al., 2005). A isso tudo, acrescenta-se os avanços da medicina que possibilita a comprovação da capacidade de cura de determinadas plantas, o que garante segurança na utilização das mesmas como medicamento. Devese ressaltar que as drogas derivadas de plantas também podem ser prejudiciais para o organismo, uma vez que é constituída por centenas de compostos e alguns são muito tóxicos. Todavia os efeitos adversos dos agentes fitoterápicos são menores, quando comparado com o das drogas sintéticas (Calixto, 2000).

O gênero Piper (família Piperaceae) inclui um grande número de espécies conhecidas popularmente como Pariparoba muito utilizadas no tratamento de diversas doenças, cuja importância econômica e comercial se deve à produção de óleos essenciais de interesse na indústria farmacêutica e de inseticida (Corrêa, 1984; Maia et al., 1987; Silva; Machado, 1999; Santos et al., 2001; Mesquita et al., 2005). Cerca de 700 espécies pertencentes ao gênero Piper estão distribuídas nos dois hemisférios, tanto em regiões tropicais como em subtropicais (Parmar et al., 1997). Na Jamaica, de 11 espécies estudadas $P$. aduncun (L) e P. hispidum (SW) foram listadas como remédio para estômago e repelente de insetos. P. amalago (L.), nativa do México, é usada para aliviar dores no estômago e como antiinflamatório e $P$. marginatum nativa do Brasil mostrou atividade antiinflamatória (Falcão et al 2005). Raízes e frutos de P. chaba (Hunter) têm muitas aplicações na medicina, dentre elas no tratamento de asma, bronquite e dores abdominais (Parmar et al., 1997) mas, um estudo com P. nigrum dos Estados Unidos da América, não mostrou atividade antidiabética in vitro (Barbosa-Filho et al., 2005).

A Piper regnellii var. pallescens apresenta amplo espectro de atividade antimicrobiana (bactérias
Gram-positivas, Gram-negativas e fungos leveduriformes) (Holetz et al., 2002; Pessini et al 2003a). Estudos fitoquímicos da raíz mostraram que esta espécie apresenta um acúmulo de vários fenilpropanóides e neolignanas dihidrobenzofuranicas incluindo o conocarpano como composto majoritário (Benevides etal., 1999; Pessini etal., 2005). Recentemente, Luize et al. (2005) demonstraram que extratos brutos de folhas de $P$. regnellii (Miq.) C. DC. var. regnellii apresentam atividade antileishmania e antitripanossoma.

No presente estudo foi avaliada a atividade antileishmania de extratos brutos e frações obtidas de folhas de $P$. regnellii var. pallescens em formas promastigota de $L$. amazonensis. A atividade citotóxica também foi verificada em macrófagos J774G8 através da técnica da Sulforodamina B.

\section{MATERIAL E MÉTODOS}

\section{Obtenção do extrato bruto e frações}

As folhas de $P$. regnellii var pallescens foram coletadas no Horto de plantas medicinais "Prof". Irenice Silva" do campus da Universidade Estadual de Maringá (UEM). A espécie vegetal foi identificada por MSc. Marília Borgo do Departamento de Botânica da Universidade Federal do Paraná (UFPR) e, uma exsicata foi depositada como documento taxonômico no Herbário da UEM, sob número de registro HUM 8392.

O material coletado foi seco em estufa de ar circulante (Quimis $^{\circledR}$, modelo Q-31) a temperatura de $40^{\circ} \mathrm{C}$, durante $72 \mathrm{~h}$ e moído em moinho de facas (Tecnal Marconi $^{\circledR}$, modelo TE 048), acondicionado e armazenado em local seco ao abrigo da luz.

As folhas secas e moídas foram extraídas com solução hidroalcoólica a $90 \% \mathrm{v} / \mathrm{v}$, na proporção 1:10 (material vegetal:solvente). $O$ extrato foi filtrado e concentrado sob pressão reduzida em evaporador rotatório. A parte solúvel em água recebeu a denominação de extrato bruto fase aquosa (EBA) e a parte insolúvel, solubilizada em acetato de etila, denominada de extrato bruto fase acetato (EBAcOEt). Os extratos foram armazenados em frascos fechados e mantidos a $-20^{\circ} \mathrm{C} \mathrm{em}$ freezer.

Os extratos brutos (fase aquosa e fase acetato $90 \%$ ) foram submetidos aos ensaios biológicos. O EBAcOEt, que apresentou maior atividade antileishmania, foi submetido ao fracionamento por Cromatografia em Coluna (CC) de adsorção sob pressão reduzida, utilizando diferentes sistemas eluentes, sendo eles: hexano, clorofórmio, clorofórmio:acetato de etila 95:5, clorofórmio:acetato de etila 90:10, clorofórmio: acetato de etila 50:50, acetato de etila, acetona, metanol, metanol:água, cujas frações foram denominadas de FHex, FClo, FCA5, FCA10, FCA50, FAac, Face, FMet, FMea, respectivamente. Estas foram posteriormente submetidas aos ensaios biológicos. 


\section{Manutenção de Leishmania amazonensis}

Formas promastigotas de Leishmania amazonensis (cepa WHOM/BR/75/JOSEFA) foram cultivadas em meio Warren (Warren, 1960) pH 7.2, suplementado com $10 \%$ de soro fetal bovino inativado (SFB - Cultilab, Campinas) a $28^{\circ} \mathrm{C}$. As amostras foram mantidas através de passagens semanais no mesmo meio.

\section{Atividade antipromastigota}

Para avaliar o efeito dos extratos brutos e frações obtidas de folhas de $P$. regnelli var pallescens no crescimento das formas promastigotas de L. amazonensis, estes foram dissolvidos em dimetilsulfóxido (DMSO), obtendo-se uma solução estoque de $10.000 \mu \mathrm{g} / \mathrm{mL}$. Em seguida foram realizadas diluições em meio de Warren para obter concentrações finais de 1000, 500, 100 e $10 \mu \mathrm{g} /$ $\mathrm{mL}$. Nestas foram adicionados os protozoários com $72 \mathrm{~h}$ de cultivo (inóculo inicial de $1 \times 10^{6}$ céls $/ \mathrm{mL}$ ). Os ensaios foram realizados em microplacas de 24 poços, incubadas a $28{ }^{\circ} \mathrm{C}$. Após $72 \mathrm{~h}$, o crescimento foi avaliado através da contagem dos protozoários em câmara de Neubauer. Anfotericina B utilizado como controle positivo foi obtido de Cristália Produtos Químicos Farmacêuticos Ltda, Itapira, São Paulo, Brasil.

\section{Ensaio de citotoxicidade pelo método colorimétrico da sulforodamina B}

Cem microlitros de uma suspensão de macrófagos J774G8 (5 x $10^{5}$ céls/mL em RPMI 1640) foram semeados em placas de 96 poços incubados a $37^{\circ} \mathrm{C}$ com tensão de $5 \%$ de $\mathrm{CO}_{2}$. Após $24 \mathrm{~h}$ de incubação foram adicionadas $100 \mu \mathrm{L}$ das várias concentrações das soluções do extrato bruto e frações das folhas de P. regnellii e incubadas por mais $48 \mathrm{~h}$. $\mathrm{O}$ crescimento celular foi avaliado através do método colorimétrico de sulforodamina B previamente descrito (Skehan et al., 1990) com poucas modificações. As células foram fixadas com $50 \mu \mathrm{L}$ de ácido tricloroacético a $10 \%$ em temperatura de $4^{\circ} \mathrm{C}$ por $1 \mathrm{~h}$, lavadas 5 vezes com água corrente e secas. Em seguida foram adicionados $50 \mu \mathrm{L}$ de solução de sulforodamina $\mathrm{B}(0,4 \% \mathrm{p} / \mathrm{v}$ em de ácido acético aquoso $1 \%$ ) em cada poço e a placa foi incubada por $30 \mathrm{~min}$ a $4^{\circ} \mathrm{C}$ ao abrigo da luz. O corante foi removido lavando-se os poços 4 vezes com ácido acético $1 \%$. Para avaliação do crescimento celular foram adicionados $150 \mu \mathrm{L}$ de Tris base a $10 \mathrm{mM}$ em cada poço, a placa foi agitada por 15 min e a leitura realizada em leitor de ELISA (Bio-Tek FL-600 Microplate Fluorescence Reader), em densidade óptica (DO) de $550 \mathrm{~nm}$.

\section{Análise estatística}

Os dados foram analisados através da Análise de Variância (ANOVA) seguido pelo teste de Tukey. O nível de significância aceito foi de $\mathrm{p} \leq 0,05$.

\section{RESULTADOS E DISCUSSÃO}

No presente estudo foram avaliadas as atividades dos extratos brutos e suas frações, obtidas a partir de folhas de Piper regnellii var pallescens, sobre formas promastigota de L. amazonensis cultivada em meio de Warren a $28{ }^{\circ} \mathrm{C}$ durante $72 \mathrm{~h}$. O efeito do extrato bruto hidroalcoólico fase aquosa e fase acetato de etila no crescimento de formas promastigotas de $L$. amazonensis está representado na Figura 1. O EBAcOEt apresentou maior atividade sobre L. amazonensis com uma Concentração Inibitória $50 \%\left(\mathrm{IC}_{50}\right)$ de $30 \mu \mathrm{g} / \mathrm{mL}$, quando comparado com o EBA ( $\mathrm{IC}_{50}$ de $167 \mu \mathrm{g} / \mathrm{mL}$ ). Por apresentar um efeito antileishmania maior o EBAcOEt foi submetido ao processo de fracionamento utilizandose uma coluna cromatográfica de adsorção sob pressão reduzida.

A Figura 2 mostra os resultados da atividade antileishmania das 9 frações (FHex, FClo, FCA5, FCA10, FCA50, FAac, Face, FMet, FMea) obtidas a partir do EBAcOEt. As frações FHex e FClo foram as que apresentaram melhor atividade com um $\mathrm{IC}_{50}$ de 21,5 e $32,0 \mu \mathrm{g} / \mathrm{mL}$, respectivamente. Por outro lado as frações de polaridade crescente (FCA5, FCA10, FCA50, FAac, Face, FMet, FMea) apresentaram uma atividade inibitória inferior a do EBAcOEt com um $\mathrm{IC}_{50}$ de 176,5, $188,2,133,3,123,5,>1.000,>1.000$ e $>1.000 \mu \mathrm{g} / \mathrm{mL}$, respectivamente. O DMSO na concentração máxima utilizada (1\%) não interferiu no crescimento do parasita. Anfotericina B foi utilizada como controle positivo contra L. amazonensis e apresentou um $\mathrm{IC}_{50}$ de $0,060 \mu \mathrm{g} / \mathrm{mL}$ (dados não mostrados).

Em um estudo realizado com formas epimastigotas de Trypanonosoma cruzi (Chagas), Luize et al. (2002) verificaram que o extrato bruto fase acetato de etila, obtido a partir de folhas de Piper regnellii, apresenta $\mathrm{IC}_{50}$ de $54,0 \mu \mathrm{g} / \mathrm{mL}$ e a fase aquosa $\mathrm{IC}_{50}>500 \mu \mathrm{g} / \mathrm{mL}$. Similarmente aos resultados obtidos no presente trabalho, as frações menos polares do EBAcOEt apresentaram maior atividade tripanocida. As frações $\mathrm{FHex}$ e FClo, a uma concentração de $100 \mu \mathrm{g} / \mathrm{mL}$, foram capazes de inibir o crescimento das formas epimastigotas em 92,2 e 86,9 $\%$, respectivamente.

Diversos estudos têm demonstrado a atividade biológica das espécies de plantas pertencentes ao gênero Piper. Extrato de folhas de P. aduncum apresenta atividade antigonorréica (Cáceres et al., 1995), extrato de $P$. methysticum apresenta atividade antifúngica (Locher et al., 1995) e extrato clorofórmio de P. sarmentosum demonstra considerável efeito antimalárico (Rahman et al., 1999). Pessini et al. (2003b) relataram uma forte atividade de $P$. regnellii sobre Staphylococcus aureus e Bacillus subtilis, uma moderada atividade em Pseudomonas aeruginosa e uma fraca atividade em Escherichia coli. 


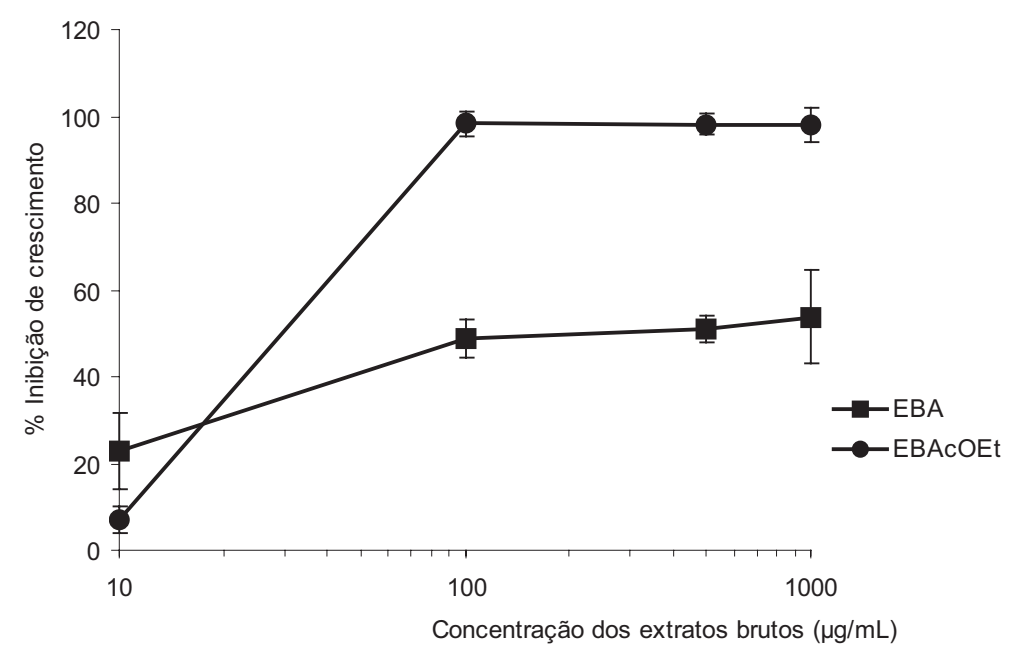

Figura 1. Efeito dos extratos brutos (EBA e EBAcOEt) obtidos a partir de folhas de Piper regnellii sobre formas promastigota de Leishmania amazonensis cultivadas durante $72 \mathrm{~h}$ nas concentrações de 10, 100 e $1000 \mu \mathrm{g} / \mathrm{mL}$. Os resultados foram realizados em duplicata e representam a média de 2 experimentos independentes.

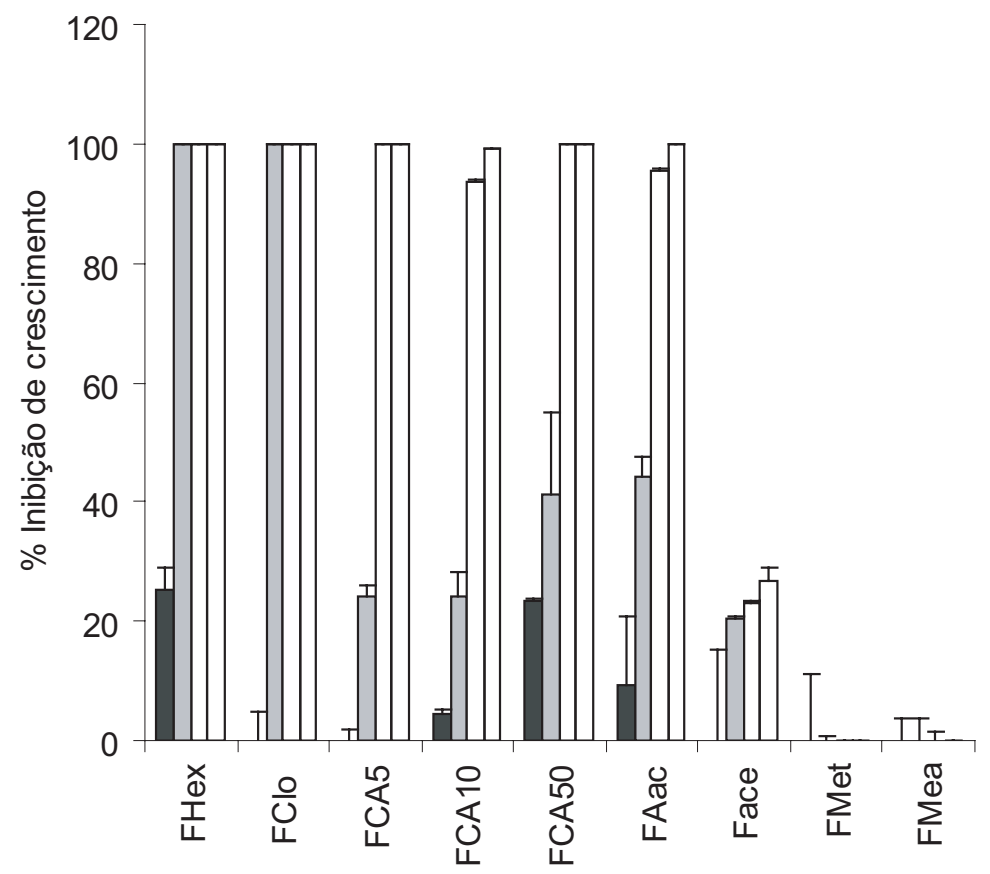

$10 \mu \mathrm{g} / \mathrm{mL} \square 100 \mu \mathrm{g} / \mathrm{mL} \square 500 \mu \mathrm{g} / \mathrm{mL} \square 1000 \mu \mathrm{g} / \mathrm{mL}$

Figura 2. Efeito das frações obtidas a partir do extrato bruto (EBAcOEt) de Piper regnellii sobre formas promastigota de Leishmania amazonensis cultivadas durante $72 \mathrm{~h}$ nas concentrações de 10, 100, 500 e $1000 \mu \mathrm{g} / \mathrm{mL}$. Os valores estão expressos em média (* estatisticamente diferente do controle - ANOVA seguida pelo teste de Tukey $\mathrm{p} \leq 0,05)$. Controle positivo: Anfotericina $\mathrm{B}\left(\mathrm{IC}_{50}=0,060 \mu \mathrm{g} / \mathrm{mL}\right)$. 
Tabela 1. Valores de $\mathrm{CC}_{50}$ para macrófagos $\mathrm{J} 774 \mathrm{G} 8$ e $\mathrm{IC}_{50}$ para formas promastigota de Leishmania amazonensis e seus respectivos índices de seletividade (IS).

\begin{tabular}{|c|c|c|c|}
\hline & $\begin{array}{c}\mathrm{J} 774 \mathrm{G} 8 \\
\mathrm{CC}_{50}\end{array}$ & $\begin{array}{c}\text { Promastigota } \\
\text { IC }_{50} \\
\end{array}$ & IS \\
\hline & \multicolumn{2}{|c|}{$\mu \mathrm{g} / \mathrm{mL}$} & \\
\hline EBA & $>1000$ & 167 & $>5,9$ \\
\hline EBAcOEt & 272 & 30 & 9,1 \\
\hline FHex & 138 & 21,5 & 6,4 \\
\hline FClo & 1000 & 32 & 31,2 \\
\hline FCA5 & 1000 & 176,5 & 5,7 \\
\hline FCA10 & 300 & 188,2 & 1,6 \\
\hline FCA50 & 300 & 133,3 & 2,3 \\
\hline FAac & 300 & 123,5 & 2,4 \\
\hline Face & $>1000$ & $>1000$ & $\mathrm{Nd}$ \\
\hline FMet & 940 & $>1000$ & $\mathrm{Nd}$ \\
\hline FMea & $>1000$ & $>1000$ & $\mathrm{Nd}$ \\
\hline
\end{tabular}

$\mathrm{CC}_{50}=$ Concentração citotóxica para $50 \%$ do tapete celular.

$\mathrm{IC}_{50}=$ Concentração capaz de inibir em $50 \%$ o número de protozoários em cultura.

$\mathrm{IS}=\mathrm{CC}_{50} \mathrm{~J} 774 \mathrm{G} 8 / \mathrm{IC}_{50}$ promastigota

$\mathrm{Nd}=$ não determinado

Produtos naturais têm um grande potencial na pesquisa por novos e seletivos agentes para o tratamento de importantes doenças causadas por protozoários (Wright; Phillipson, 1990). Várias classes de substâncias já foram isoladas a partir de espécies do gênero Piper tais como alcalóides, lignanas, neolignanas, terpenos e ácido benzóico e têm se mostrado bioativas (Zacchino et al., 1997; Park et al., 2002; López et al., 2002; Martins et al., 2003).

Um importante critério na pesquisa de compostos ativos com potencial terapêutico contra L. amazonensis, é determinar a ausência de efeitos tóxicos nas células do hospedeiro. A citotoxicidade para macrófagos J774G8 e para protozoários foi comparada utilizando o índice de seletividade (IS), que consiste na razão entre a Concentração Citotóxica 50\% $\left(\mathrm{CC}_{50}\right)$ para células $\mathrm{J774G8}$ e $\mathrm{IC}_{50}$ para protozoários. Os resultados de IS apresentados na Tabela 1 mostram que o EBAcOEt e a FHex são 9,1 e 6,4 vezes menos tóxicos para as células J774G8 do que para os protozoários. Resultado interessante também foi obtido com a fração FClo que apresentou atividade antileishmania semelhante ao do EBAcOEt, porém com um IS de aproximadamente 3,4 vezes maior.

Os resultados do presente trabalho demonstram que o extrato e frações obtidas de folhas de $P$. regnellii var pallescens apresentam atividade antileishmania. Estudos fitoquímicos e biológicos estão sendo realizados em nosso laboratório com a $P$. regnellii var pallescens para o isolamento e identificação de substâncias bioativas em L. amazonensis a partir das frações que apresentaram boa atividade.

\section{AGRADECIMENTOS}

Ao Conselho Nacional de Desenvolvimento Científico e Tecnológico - CNPq, Capacitação de Aperfeiçoamento de Pessoal de Nível Superior-CAPES, Financiadoras de Estudos e Projetos - FINEP, PRONEX/ Fundação Araucária e Programa de Pós-graduação em Ciências Farmacêuticas da Universidade Estadual de Maringá pelo auxílio financeiro.

\section{REFERÊNCIAS}

Barbosa-Filho JM, Vasconcelos THC, Alencar AA, Batista LM, Oliveira RAG, Guedes DN, Falcão HS, Moura MD, Diniz MFFM, Modesto-Filho J 2005. Plants and their active constituents from South, Central, and North América with hypoglycemic activity. Rev Bras Farmacogn 15: 392-413.

Benevides PJC, Sartorelli P, Kato MJ 1999. Phenilpropanoids and neolignans from Piper regnellii. Phytochemistry 52: 339-343.

Cáceres A, Menendez H, Méndez E, Cohobón E, Samoya BE, Jáuregui E, Peralta E, Carrilho G 1995. Antigonorrhoeal activity of plants used in Guatemal for the treatment of sexually transmitted disease. $J$ Ethnopharmacol 48: 85-88.

Calixto JB 2000. Efficacy, safety, quality control, marketing and regulatory guidelines for herbal medicines (phytotherapeutic agents). Braz J Med Biol Res 33:179-189.

Convit J, Ulrich M, Polegre MA, Avila A, Rodriguez N, Mazzedo MI, Blanco B. 2004. Therapy of Venezuelan patients with severe mucocutaneous or early lesions of diffuse cutaneous leishmaniasis with vaccine containing pasteurized Leishmania promastigotes 
and Bacillus calmett-guerin - Preliminary report. Mem I Oswaldo Cruz 99:57-62.

Corrêa MP 1984. Dicionário das plantas úteis do Brasil e das exóticas cultivadas, Rio de Janeiro: Empresa Nacional.

Croft SL, Coombs GH 2003. Leishmaniasis - current chemotherapy and recent advances in the search for novel drugs. Trends Parasitol 19: 502-508.

Grevelink SA, Lerner EA 1996. Leishmaniasis. J Am Acad Dermatol 34: 257-272.

Handman E 1997. Leishmania vaccines: Old and new. Parasitol Today 13: 236-238.

Herwaldt BL 1999. Leishmaniais. Lancet 354: 1191-1199.

Holetz FB, Pessini GL, Sanches NR, Cortez DAG, Nakamura CV, Dias Filho BP 2002. Screening of some plants used in Brazilian folk medicine for treatment of infectious diseases. Mem I Oswaldo Cruz 97: 10271031.

Locher CP, Burch MT, MowerHF, Berestecky J, Davis H, Van Poel B, Lasure A, Vanden DAB, Vlietinck AJ 1995. Antimicrobial activity and anti-complement activity of extracts obtained from selected Hawaiian medicinal plants. J Ethnopharmacol 49: 23-32.

López A, Ming DS, Towers GHN 2002. Antifungical activity of benzoic acid derivatives from Piper lanceaefolium. J Nat Prod 65: 62-64.

Luize PS, Morello LG, Ueda-Nakamura T, Dias Filho, BP, Cortez DAG, Nakamura CV 2002. Antitrypanosoma activity of extract obtained from plant Piper umbellatum. Rev Fitoter 2: 315.

Luize PS, Tiuman TS, Morello LG, Maza PK, Ueda-Nakamura T, Dias Filho BP, Cortez DAG, Mello JCP, Nakamura CV 2005. Effects of medicinal plant extracts on growth of Leishmania (L.) amazonensis and Trypanosoma cruzi. Rev Bras Ci Farma 41: 8594.

Maia JGS, Silva ML, Luz AIR, Zoghbi MGB, Ramos LS 1987. Espécies de Piper da Amazônia ricas em safrol. Quim Nova 10: 200-204.

Martins RC, Lago JH, Albuquerque S, Kato, MJ 2003. Trypanocidal tetrahydrofuran lignans from inflorescences of Piper solmsianun. Phytochemistry 64: 667-670.

Mesquita JMO, Cavaleiro C, Cunha AP, Lombardi JA, Oliveira AB 2005. Estudo comparativo dos óleos voláteis de algumas espécies de Piperaceae. Rev Bras Farmacogn 15: 6-12.

Michalick MSM 2003. Gênero Leishmania. In: Neves DP, Melo AL, Genaro O, Linardi PM Parasitologia humana. 10. ed. São Paulo: Atheneu, p.31-35.

Murray PR, Rosenthal KS, Kobayashi GS, Pfaller MA 2000. Microbiologia médica. Rio de Janeiro: Guanabara Koogan.

Park SB, Lee SE, Shin SC, Park JD, Ahn YJ 2002. Larvicidal activity of isobutylamides identified in Piper nigrum fruits against three mosquito species. J Agric Food Chem 50: 1866-1870.

Parmar VS, Jain SC., Bisht KS, Jain R, Taneja P, Jha A, Tyagi OD, Prasad AK, Wengel J, Olsen CE, Boll PM 1997. Phytochemistry of the genus Piper. Phytochemistry 46: 597-673.

Pessini GL, Holetz FB, Sanches NR, Cortez DAG, Dias-Filho BP, Nakamura CV 2003a. Avaliação da atividade antibacteriana e antifúngica de extratos de plantas utilizados na medicina popular. Rev Bras Farmacogn 13(Supl 1): 21-24.

Pessini GL, Dias Filho BB, Nakamura CV, Cortez DAG 2003b. Antibacterial activity of extracts and neolignans from Piper regnellii (Miq.) C. DC. var. pallescens (C. DC.) Yunck. Mem I Oswaldo Cruz 98: 11151120.

Pessini GL, Dias-Filho BP, Nakamura CV, Ferreira AG, Cortez DAG 2005. Neolignanas e análise do óleo essencial das folhas de Piper regnellii (Miq.) C. DC. var. pallescens (C. DC.) Yunck. Rev Bras Farmacogn 15: 199-204.

Rahman NNNA, Furuta T, Kojima S, Takane K, Mohd MA 1999. Antimalarial activity of extracts of Malaysian medicinal plants. J Ethnopharmacol 64: 249-254.

Rocha LG, Almeida JRGS, Macêdo RO, Barbosa-Filho JM 2005. A review of natural products with antileishmanial activity. Phytomedicine 12: 514535.

Santos PRD, Moreira DL, Guimarães EF, Kaplan MAC 2001. Essencial oil analysis of 10 Piperaceae species from the Brasilian Atlantic Forest. Phytochemistry 58: 547-551.

Sereno D, Holzmuller P, Lemesre JL 2000. Efficacy of second line drugs on antimonyl-resistant amastigotes of Leishmania infantum. Acta Trop 74: 25-31.

Silva EMJ, Machado SR 1999. Estrutura e desenvolvimento dos tricomas secretores em folhas de Piper regnellii (Miq.) C. DC. var. regnellii (Piperaceae). Rev Bras Bot 22: 117-124.

Skehan P, Storeng R, Scudeiro D, Monks A, Mcmahoin J, Vistica D, Warren JT, Bokesch H, Kenney S, Boyd MR 1990. New colorimetric cytotoxicity assay for anticancer-drug screening. J Nat Canc Inst 82: 1107.

Tewary P, Sukumaran B, Saxena S, Madhubala R 2004. Immunostimulatory oligodeoxynucleotides are potent enhancers of protective immunity in mice immunized with recombinant ORFF leishmanial antigen. Vaccine 22: 3053-3060.

Warren LG 1960. Metabolism of Schizotrypanum cruzi Chagas. I. Effect of culture age and substrate concentration on respiratory rate. J Parasitol 46: 529-39.

WHO (World Health Organization) 2002. Urbanization: an increasing risk factor for leishmaniasis. Disponível em $<$ http://www.who.int/wer $>$, acessada em fev. 2005.

Wright CW, Phillipson JD 1990. Natural products and the development of selective antiprotozoal drugs. Phytother Res 4: 127-139.

Zacchino S, Rodrígues G, Pezzenati G, Orellana G 1997. In vitro evaluation of antifungal properties of 8.O.4' neolignans. J Nat Prod 60: 659-662. 